\title{
Research on XBOM model for supporting product collaborative innovation
}

\author{
A. Zhang Yi-ying, Liu Xiao-bing \\ Faculty of Management and Economics, Dalian University of Technology, Dalian 116023, China \\ B. Mu Wei \\ CSR Qingdao Sifang Co., Ltd. Qingdao 266111, China \\ C. Bo Hong-guang \\ Faculty of Management and Economics, Dalian University of Technology, Dalian 116023, China
}

KEYWORDS: xBOM model, product collaborative innovation

\begin{abstract}
With the increasingly fierce competition among manufacturing enterprises, collaborative innovation of the products is very important. By defining the virtual part, intermediate part, outsourcing part and the relevant mapping function, it is realized that the Engineering $\mathrm{BOM}(\mathrm{EBOM})$ and Process planning $\mathrm{BOM}(\mathrm{PPBOM})$ are transformed into Manufacturing $\mathrm{BOM}(\mathrm{MBOM})$. It effectively keeps the integrity, correctness and consistency of the BOM view data.
\end{abstract}

\section{INTRODUCTION}

In manufacturing industry, Bill of Material(BOM) is needed for productions. BOM data can be used to describe the composition and relationship of the material of product. It can be also used to indicate product brochures as well, product packaging, and other relevant files of parts.

In manufacturing industry, BOM is the core basic data and important part of product digital definition ${ }^{[1]}$. BOM is the connection between enterprise product engineering design and production operation management. In different stages of product whole life cycle and in the face of different production departments, there are a variety of different meanings and uses of the BOM views. Therefore, it is of great significance to achieve the collaborative innovation of the product and ensure the integrality, correctness and consistency of BOM views data.

\section{XBOM IN THE MANUFACTURING INDUSTRY}

Typically, BOM has following several major types for different purposes to create, design and use $^{[2]}$ :

(1)Engineering BOM(EBOM).

EBOM is part bill of materials to organize and manage the production by the enterprise product design department. After design engineer has finished production design, EBOM is obtained.

(2) Process planning BOM(PPBOM)

PPBOM is process files to organize and manage the product and its related parts by the enterprise process design department. It is designed on the basis of EBOM. EBOM and PPBOM are the most original BOM in varied BOM. They condense the creative of product design engineers and process engineers. Other kinds of $\mathrm{BOM}$ are transformed by combining information from their applications on the basis of EBOM and PPBOM.

(3) Manufacturing BOM (MBOM)

MBOM is the part of BOM which need in actual manufacturing and production management process by the enterprise manufacturing department. MBOM is based on EBOM and PPBOM. It plays an important role in MRP and ERP.

(4) Quality BOM(QBOM)

Enterprise production management department and quality control department describe quality requirements, quality testing and quality control standards of self-made parts, outsourcing parts and 
purchasing parts, according to quality requirements of MBOM and PPBOM. They are used to guide the production quality control and inspection.

There are also Cost BOM(CBOM), Buying BOM (BBOM), Order BOM(OBOM) and so on in manufacturing industry.

Above-mentioned $\mathrm{BOM}$ are called $\mathrm{xBOM}$. Their generation and using are closely related to the development process of products. The various BOM is a kind of view of relationship in three dimensional space determined by the type of product.

\section{TRANSFORMATION FROM EBOM TO MBOM}

In traditional enterprise, there are all or part of above-mentioned BOM views. The department design, manufacture, store and manage all kinds of BOM views in order to meet their need. But there is no unified design and management standard in the process of BOM design and manufacture. In addition to EBOM and PPBOM can be obtained automatically by integrating with CAD and CAPP systems, other BOM views are established manually. Therefore, the correctness, integrity and consistency of the data between each BOM view can't be guaranteed. Building BOM view data is also a time-consuming and laborious task. This phenomenon has seriously affected the integration and work efficiency of product design, production and management process in enterprise, increased production costs and extended the development cycle.

According to the definition of BOM view, EBOM and PPBOM are original BOM views. They are called initial BOM views. Other BOM views are transformed based on EBOM and PPBOM basically. They are called export BOM views of EBOM and PPBOM. There must be some kind of mapping relationship between initial $\mathrm{BOM}$ views and export $\mathrm{BOM}$ views. This relationship can be expressed in the following functions:

$D_{-} B O M=f_{I \rightarrow D}($ EBOM, PPBOM $)$

In the function: $f_{t \rightarrow 0}$ is the mapping function between initial BOM views and export BOM views. D_BOM is the export BOM view. EBOM is engineering BOM. PPBOM is process planning BOM. The mapping relationship between initial $\mathrm{BOM}$ views and export $\mathrm{BOM}$ views can be identified by analyzing the transformation between various export BOM views.

MBOM is built based on the EBOM and PPBOM by manufacturing department. The transformation from EBOM and PPBOM to MBOM is the most important and the most practical in all BOM transformation. Typically, the MBOM and EBOM are different. After extensive investigation and analysis, the difference is attributed to the following three situations:

(1) Virtual part

Virtual part is the part that appears in EBOM, but doesn't manufacture and storage in the actual production. Virtual part isn't recorded in PPBOM.

(2) Intermediate part

Intermediate part is the part that doesn't appear in PPBOM. But it is necessary to manufacture and storage because of process requirements in the actual production. Intermediate part is recorded in PPBOM. It's production type is self-made part.

(3) Outsourcing part

Outsourcing part is the part itself or all its child parts are outsourced. Outsourcing part is only described the production type as outsourcing processing.

Figure 1 describes the above three special parts. Letters represent part ID. Numbers represent the number of assemblies that are relative to the parent part. 


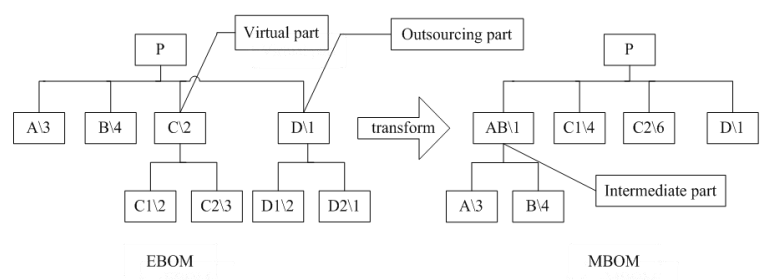

Figure 1. Transformation from EBOM to MBOM.

The above three situations can be handled according to the following three mapping relationships:

(1) Virtual part mapping relationship

If there is virtual part in the EBOM, its quantity relations in the EBOM will be moved to the parent part of the virtual parts in the MBOM. The processing steps are as follows:

(1) find out all the virtual parts in EBOM and PPBOM, push them onto the stack named parts stack; (2) pop an element to parts from the parts stack when the parts stack is nonempty, otherwise, go to step (9); (3) find the parent parts in EBOM to identify father_ID and its assembly quantity np; (4)find all child parts of the parts in EBOM (including product identification child_ID and assembly quantity nc and other related information), enter them in the stack named child stack; (5) pop an element to child from the child stack when the child_stack is nonempty, otherwise, go to step (B); (G) change the parent part identification of the child to father ID, assembly quantity is the product of np and nc; (7) go to step (5); (4) go to step (2); $y_{\text {go }}$ to finish.

Structure mapping mathematical model can be constructed:

Definition 1: node $\mathrm{p}$.

Node $\mathrm{p}$ is a set of characteristics of all nodes, which are defined and described.

$\mathrm{P}=\left\{\mathrm{c}_{1}, \mathrm{c}_{2}, \ldots, \mathrm{c}_{\mathrm{s}}\right\}$

Definition 2: ordered pair $r$.

The $\mathrm{r}$ is a triple, the first element represents the parent node, the second element represents the child node, the third element indicate the number of them.

$\mathrm{r}=<\mathrm{p}_{1}, \mathrm{p}_{2}, \mathrm{z}>$

It means: $\mathrm{P}_{1}$ is the parent node of $\mathrm{P}_{2}$, and $\mathrm{P}_{1}$ is composed of $\mathrm{z} \mathrm{P}_{2}$. If $\mathrm{r}=<\mathrm{p}_{1}, \mathrm{p}_{2}, 0>$,it's empty.

Assuming that $\mathrm{p}$ is a virtual part, $\mathrm{p}_{\mathrm{f}}$ and $\mathrm{p}_{\mathrm{c}}$ are the father part and the child part respectively. In EBOM and PPBOM, $r_{1}=<p_{f}, p, n_{p}>, r_{2}=<p, p_{c}, n_{c}>$, then delete the virtual part $p$ in $M B O M, r=<p_{f}$, $\mathrm{p}_{\mathrm{c}}, \mathrm{n}_{\mathrm{p}} * \mathrm{n}_{\mathrm{c}}>$

(2) Intermediate part mapping relationship

If there is a intermediate part, bill of materials and assembly relation information are added in MBOM, according to the related information of the intermediate part in PPBOM and the related information of father part and child part in EBOM. The processing steps are as follows:

(1) find out all the intermediate parts in EBOM and PPBOM, push them onto the stack named parts_stack; (2) pop an element to parts from the parts_stack when the parts_stack is nonempty, otherwise, go to step (9) ; (3) find the parent parts in PPBOM to identify father_ID and its assembly quantity $\mathrm{np}$, add the record that parent part identification is father_ID and child part identification is parts, its assembly quantity is np; (4) find all child parts in PPBOM and assembly quantity nc, enter them in the stack named child_stack; (5) pop an element to child from the child_stack when the child_stack is nonempty, otherwise, go to step (G); (G) find the record that the parent part identification is father_ID and child part identification is parts. Its assembly quantity is original assembly quantity minus the product of $\mathrm{np}$ and nc. If the modified assembly quantity is 0 , delete the record; (7) go to step (5); (G) go to step (2); (ㄴ) go to finish. 
Assuming that $\mathrm{p}$ is an intermediate part, $\mathrm{p}_{\mathrm{f}}$ and $\mathrm{p}_{\mathrm{c}}$ are the father part and the child part respectively when $p$ is added in MBOM. In EBOM and PPBOM, $r_{0}=<p_{f}, p_{c}, n_{p c}>, r_{1}=<p_{f}, p, n_{p}>$, $r_{2}=<p, p_{c}, n_{c}>$, then add the intermediate part $p$ in MBOM, $r=<p_{f}, p_{c}, n_{p c}-n_{p} * n_{c}>$. If $n_{p c}-n_{p} * n_{c}=0$, delete the record.

(3) Outsourcing part mapping relationship

In the MBOM, only the outsourcing parts themselves are described, the lower parts material information of the outsourcing parts is not described. So all of the outsourcing parts are at the leaf nodes of the BOM tree. Its process is a breadth-first or depth-first search process. The process ignores the processing of the mapping relationship. According to the above three kinds of mapping relationship, the transformation function from EBOM to MBOM is:

$$
M B O M=f_{3}\left(f_{2}\left(f_{1}(E B O M, P P B O M)\right)\right)
$$

In the function: $f_{1}$ is virtual part mapping relationship; $f_{2}$ is intermediate part mapping relationship; $f_{3}$ is outsourcing part mapping relationship, MBOM is manufacturing BOM. The transformation from EBOM to MBOM is divided into two processes. First, get the original MBOM by copying all of the EBOM. Second, the initial MBOM processing is divided into three steps, according to the above functions: (1) Traverse the various nodes of EBOM by the way of breadthfirst or depth-first. For each node, find whether there is a description of its parts in PPBOM. If there is no description, handle it as virtual part mapping relationship and obtain the transitional MBOM without the virtual part information. (2) Find the self-made part described in the PPBOM but not described in the transitional MBOM in step one. Handle it as intermediate part mapping relationship, obtain the transitional MBOM that deletes the virtual part information and adds intermediate part information. (3) Find the outsourcing part described in the PPBOM, handle it as outsourcing part mapping relationship in the transitional MBOM in step two. Finally obtain a correct, integral and consistent MBOM that meets the requirements of production management and control. This MBOM has an important position in ERP and MRPII. It is an important file for the production planning and scheduling control.

\section{TRANSFORMATION TECHNOLOGY OF BOM VIEW AND ITS APPLICATION}

In the project of CSR Qingdao Sifang Co., Ltd., the transformation technology between the BOM view is widely used. It achieved a rapid establishment of MBOM, greatly reducing the data entry and accuracy testing. The consistency, integrity and correctness of BOM data are effectively guaranteed. In the implementation process, the following measures and data structures are used:

EBOM and MBOM are described by using two relationships ${ }^{[3,4]}$ : father-son assembly relationship and nature relationship of parts. The parts assembly and nature relationship of parts are described only once. It can reduce the redundancy and improve the consistency of the BOM data. PPBOM is described mainly through the processing procedure and production type of parts.

The practical application shows that the enterprise doesn't need to keep all BOM views in the case of the product structure is not very complex ${ }^{[5]}$. In addition to EBOM and PPBOM, other BOM views can be quickly obtained through computer processing. This will reduce the incidence of BOM data errors.

\section{CONCLUSIONS}

Productions information management is an important aspect of production and development in the 21 st century. Management model of product data is based on the 'integration thought', which makes it easy to get all kinds of information related to the product in the whole enterprise ${ }^{[6]}$. Therefore, in the process of advanced design and manufacturing, the integrity, correctness and consistency of $\mathrm{xBOM}$ data, and establishment of $\mathrm{xBOM}$ is rapidly of great significance. By the definition of virtual part, intermediate part, outsourcing part and the corresponding processing rule, the transformation from EBOM and PPBOM to MBOM is achieved. The transformation technology among BOM views can meet the product digital definition, classification design and system integration requirements for BOM. It support the collaborative innovation of the product. The practical application shows that the transformation technology between BOM views is effective and feasible. 


\section{ACKNOWLEDGMENTS}

This research was financially supported by a project supported by National Science and Technology Support Program, China(2015BAF08B02) and the State Key Program of NSFC(71172137).

\section{REFERENCE}

[1] BO Hong-guang, LIU Xiao-bing, LV Yan-xia. "Research on xBOM Process Integration in Iron and Steel Enterprise", Industrial Engineering and Management, vol. 14, Apr. 2009, PP.76-80.

[2] WEI Zhiqiang. Digital product definition and research on the key technologies of product data management under distributed environment. Beijing: T singhua Univ., 2000.

[3] SHI Shuangyuan, ZHANG Jinlong, CAI S huqing. "Conversion model and its algorithm between level code BOM and pointer code BOM". Jour. Huazhong Univ. of Sci. \& Tech. , vol 28, Nov. 2000, PP. 67-69.

[4] SHI S huangyuan, ZHANG Jinlong, CAI Shuqing. "Pointer BOM in MRPII \& ERP and its algorithm". Jour. Huazhong Univ. of Sci. \& Tech., vol 28, Nov. 2000, PP. 64 - 66.

[5] WANG Yang, LIU Xiao-bing, HUANG Xue-wen. "Integration framework of semantic BOM knowledge for cloud manufacturing". Application Research of Computers, vol 30, 2013, PP. 20682071.

[6] REZAYAT M. "Some aspects of product and process development in the 21st century". Computer - Aided Design, vol 32, 2000, PP. 83. 